\title{
When we sneeze, does the immune system catch a cold?
}

\section{Viral infections may have important effects on the body's response to bacteria and other viruses}

The presence of pathogens is often insufficient alone to produce illness. Many healthy people carry pathogenic bacteria (such as Neisseria meningitidis or Streptococcus pneumoniae) in the upper respiratory tract yet only a few develop invasive disease. Among the factors that convert carriage to disease is coinfection with common cold viruses. This interaction was recently studied in Chad, where about one in three children were carriers of $N$ meningitidis, probably imported from Mecca by returning pilgrims. An epidemic of meningococcal meningitis occurred the year after the pilgrimage, and at its peak 141 patients were admitted to one hospital in a single day. Some 4500 cases of meningococcal disease occurred in the city of N'Djamena - an attack rate of $0.9 \%$. Careful attempts were made to isolate respiratory pathogens from 62 patients with meningococcal meningitis and from 62 matched controls. Positive isolations of Mycoplasma spp, adenovirus, respiratory syncytial virus, or parainfluenza viruses were made in $47 \%$ of the patients with meningitis and $11 \%$ of the control group, giving a matched odds ratio of 23 ( $95 \%$ confidence interval $3 \cdot 1$ to 170$){ }^{1}$

The association between infection with a respiratory virus and meningococcal disease has also been noted in Britain. An outbreak of influenza A during November and December of 1989 was followed about two weeks later by a fourfold increase of positive isolations of $N$ meningitidis in England and Wales. Serum was collected from 43 patients infected with $N$ meningitidis, and in $12(28 \%)$ there was evidence of recent infection with influenza; only $9 \%$ of the matched controls had similarly raised levels of antibodies to influenza. Patients were also questioned about the occurrence of symptoms of influenza before the onset of meningococcal disease: $60 \%$ (49/81) remembered such an illness compared with $48 \%$ (49/102) of controls. ${ }^{2}$ This study may be criticised for not showing data from patients infected with $N$ meningitidis before the influenza epidemic as a severe bacterial infection might possibly induce a non-specific increase in serum antibody to an irrelevant pathogen. Questionnaires that rely on memory of events that occurred some months earlier are notoriously unreliable, but these studies from Africa and Britain, together with circumstantial reports from North America, ${ }^{3}$ indicate that respiratory viruses probably enhance dissemination of meningococcal infections.

The recognition that common upper respiratory infections act as cofactors for bacterial infection is not new. ${ }^{+}$Bacterial otitis media is frequently preceded by a cold, and infection with influenza often promotes bacterial pneumonia, ${ }^{5}$ perhaps by enhancing bacterial adhesion to cells in the respiratory tract. A well documented apparent interaction of this sort was published in 1960: it described "cloud babies"-babies surrounded by clouds of staphylococci-who cause outbreaks of staphylococcal infection in neonatal units. In two of the three such outbreaks each cloud baby was found to be coinfected with echovirus or adenovirus. By contrast, nonshedding carriers of staphylococci in the same neonatal units were found not to be carrying viruses in the respiratory tract. ${ }^{6}$

Cold viruses may also modify responses to other viral agents. In one recent study of young children receiving standard measles-mumps-rubella vaccine 10 out of $47(21 \%)$ with symptoms of upper respiratory infection showed no detectable antibody response to the measles component of the vaccine compared with only one out of the $51(2 \%)$ asymptomatic controls. ${ }^{7}$ Current advice to doctors and parents is that afebrile colds do not contraindicate vaccination, and, as the accompanying editorial points out, ${ }^{8}$ it would be premature to change this advice as failure to vaccinate (owing to intercurrent illness) has contributed to measles outbreaks in the past. ${ }^{9}$ Krober et al used a commercially available but non-standard indirect test for measles antibodies, and the results need to be repeated with conventional enzyme linked immunosorbent assays (ELISA) and virus neutralisation assays. It should also be noted that $79 \%$ of those with colds developed a good serological response to measles and that the response to other components of the vaccine were normal (in the few children in whom they were measured).

Another classic example of viral interaction is the absence of a measles rash around chickenpox lesions in children with simultaneous infections. ${ }^{10}$ Infection with HIV now provides some of the most florid cases of dual infection, owing to its effects on CD4+ T cells. A more direct interaction is seen in the requirement of the $\delta$ hepatitis virus (an RNA virus) for simultaneous infection with hepatitis $B$ virus (a DNA virus) in order to reproduce. Another esoteric but interesting example is of infection with lactate dehydrogenase elevating virus. This virus is normally benign but becomes neurotropic in animals that carry a retrovirus that seems to display a receptor for lactate dehydrogenase elevating virus in the central nervous system. This interaction induces progressive degeneration of anterior horn cells, producing a disease with some similarity to motor neurone disease. ${ }^{11}$

With few exceptions the mechanisms by which dual 
infections enhance disease are open to speculation. Some effects of dual infection may simply be due to production of interferon, but the true mechanisms of interaction remain largely unknown. Physical effects at the epithelial or mucosal site of entry may be important, and antigens may compete for the attention of the immune system after entry into the host. Most pathogens have probably evolved elaborate molecular methods of interfering with the immune response, and the well adapted host has probably got molecular counterstratagems which might be relatively specific to a single infecting agent. The complexities of the processing and presentation of antigens are only recently becoming clear, while the checks and balances present within even the currently known network of immune cytokines are so complex that effects in intact lymphoid organs are almost impossible to predict.

The sheer number of clinical and subclinical infections that occur in the first years of life makes simultaneous infections common. In the laboratory we strive to study infections by single agents, but this event is probably rare even in the best controlled experiments. In real life infections do not occur in isolation and need to be viewed in the context of the previous and concurrent experience of the immune system. The potential complexity of dual infections is daunting, but it needs to be taken into account if we are to unravel the immense variations in disease.

Wellcome Trust Senior Research Fellow in

P J M OPENSHAW

Clinical Sciences,

Respiratory Unit,

St Mary's Hospital Medical School,

London W2 IPG

1 Moore PS, Hierholzer J, DeWitt W, Gouan K, Dioré D, Lippeveld T, et al. Respiratory viruses and mycoplasma as cofactors for epidemic group A meningococcal meningitis. JAMA $1990 ; 264: 1271-5$.

2 Cartwright KAV, Jones DM, Smith AJ, Stewart JM, Kaczmarski EB, Palmer SR. Influenza A and meningococcal disease. Lancet 1991;338:554-7.

3 Harrison LH, Armstrong CW, Jenkins SR, Harmon MW, Ajello G, Miller GB, et al. A cluster of meningococcal disease on a school bus following epidemic influenza. Arch Intern Med 1991;151:1005-9.

4 Nichol KP, Cherry JD. Bacterial-viral interrelations in respiratory infections of children. $N$ Englf Med 1967;277:667-72.

5 Loosli CG. Influenza and the interaction of viruses and bacteria in the respiratory tract. Medicine 1973;52:369-84.

6 Eichenwald HF, Kotesvalov O, Fasso LA. The "cloud baby": an example of bacterial-viral interaction. Am F Dis Child 1960;100:161-73.

7 Krober MS, Stracener CE, Bass JW. Decreased measles antibody response after measles-mumpsrubella vaccine in infants with colds. FAMA 1991;265:2095-112.

8 Peter G. Measles immunisation: recommendations, challenges, and more information. FAMA 1991;265:2111-2.

9 Hutchins SS, Escolan J, Markowitz LE, Hawkins C, Kimbler A, Morgan RA, et al. Measles outbreak among unvaccinated preschool-aged children: opportunities missed by health care providers to administer measles vaccine. Pediatrics 1989;83:369-74

10 Knight V, Fleet WF, Lang DJ. Inhibition of measles rash by chickenpox. $7 A M A$ 1964;188:690.

11 Contag $\mathrm{CH}$, Harty JT, Plagemann PGW . Dual virus etiology of age-dependent poliomyelitis of mice. A potential model for human motor neuron diseases. Microb Pathog 1989;6:391-401.

\section{The rise and fall of chorionic villus sampling}

\section{Midtrimester amniocentesis is usually preferable}

For prenatal diagnosis, which is better: chorionic villus sampling in the first trimester or amniocentesis in the second? Two years ago a large Canadian study comparing the two procedures suggested that fetal losses were slightly higher in those women randomised to chorionic villus sampling. ${ }^{.}$Now the even larger Medical Research Council European trial has confirmed this: of women allocated to amniocentesis $91 \%$ had a live baby who survived, significantly more than the $86 \%$ who were allocated to chorionic villus sampling. ${ }^{2}$

Much of this difference was accounted for by significantly higher fetal loss before 28 weeks' gestation and more pregnancies terminated for chromosomal anomalies in the women allocated to chorionic villus sampling. (Some of the pregnancies in the chorionic villus sampling group were terminated for chromosomal abnormalities that may have been lethal by the time of the amniocentesis; if so the quoted difference in miscarriage rates underestimates the true difference in miscarriages related to the procedure.) Experienced operators can achieve much lower rates of fetal loss after both transabdominal and transcervical chorionic villus sampling than were reported in these studies, ${ }^{3}$ and some improvement in differential loss rates was noted during the Medical Research Council trial itself. On the basis of these two trials, however, we must conclude that chorionic villus sampling is riskier than amniocentesis for the fetus.

Of possibly greater concern to parents is a putative link between placental biopsy and limb abnormalities. One baby in the chorionic villus sampling group in the Medical Research Council trial was delivered with an oromandibularlimb hypogenesis syndrome-one of four cases already reported from Oxford. ${ }^{4}$ This prompted many subsequent reports of limb defects in association with chorionic villus sampling, including three further cases of combined limb and facial abnormalities, from Italy, ${ }^{5}$ China,${ }^{6}$ and Germany. ${ }^{7}$ As the expected incidence of this condition is less than one in 100000 it seems quite likely that we are dealing with a real effect rather than random clustering, but this cannot be proved in the absence of complete ascertainment of all congenital anomalies following chorionic villus sampling (the numerator) and a reliable estimate of all procedures carried out (the denominator).

In addition to the risk of the syndrome of limb and facial abnormalities, the risk of transverse limb defects in general was found to be increased in a case-control study from Italy, where the popularity of chorionic villus sampling makes this method of analysis possible. ${ }^{5}$ Three reports totalling 25000 procedures were published in response to these data, and 13 cases of transverse limb defects were found. ${ }^{7.9}$ Based on the Poisson distribution for rare events, the probabilities of obtaining this (or a more extreme) result are 0.008 and 0.07 for baseline incidences of $1.8^{10}$ and $3.3^{5}$ in 10000 respectively. Publication bias is unlikely as there were no significant associations within individual series and authors therefore published their results as reassuring evidence. The apparent causal association between chorionic villus sampling and limb reduction defects is greatest before nine completed weeks of pregnancy, suggesting that the embryo should be left alone during the phenocritical stage of development. ${ }^{11}$

Chorionic villus sampling retains its great advantage over midtrimester amniocentesis by providing early results, although this potential benefit is not always realised. This is because the test throws up ambiguous mosaic results more often than amniocentesis, and as three quarters of these are confined to the placenta subsequent confirmatory tests may be required in later pregnancy. ${ }^{12}$ Both chorionic villus sampling and amniocentesis may miss small chromosomal deletions, and both produce very few false negative results for trisomy. With chorionic villus sampling, however, this is achieved only 